\section{Disinfection and Sterilization of Prion-Contaminated Medical Instruments}

To the Editor-In the February 2010 issue of the journal, under "SHEA Guideline," Rutala and Weber ${ }^{1}$ recommended sterilization options for decontaminating neurosurgical instruments exposed to the infectious agents of transmissible spongiform encephalopathies (TSE agents; prions). The recommendations of Rutala and Weber ${ }^{1}$ are contrary to those endorsed by many other experts in the field, including authors of some studies cited in their article. Conflicting TSE decontamination protocols have been proposed in the past because of concerns about the unusual resistance of infectivity to conventional sterilization methods, the paucity of appropriate laboratory and epidemiologic data, and the difficulties associated with conducting inactivation studies more relevant to clinical settings. To address the inconsistent recommendations, the World Health Organization (WHO), in 1999, convened an international consultation of prominent prion disease researchers, including experts in agent inactivation, biology, and epidemiology. ${ }^{2}$ The consultation offered various options appropriate for hospitals in developed and developing countries, with a consensus recommendation to inactivate prions, whenever possible, by means of immersion in 1-2 $\mathrm{N}$ $\mathrm{NaOH}$, followed by heating in a gravity displacement autoclave at $121^{\circ} \mathrm{C}$ for 30 minutes and cleaning and sterilization by conventional means. Rutala and Weber's ${ }^{1}$ option 1 -initially cleaning the instruments and then autoclaving at $134^{\circ} \mathrm{C}$ for 18 minutes-was viewed by the WHO consultants as the least effective procedure and was accompanied by a warning that it was likely to leave residual infectivity in some circumstances and should be used only in those clinical situations where limited resources preclude using more stringent options. $^{2}$

The recommendation of Rutala and Weber ${ }^{1}$ seems to be based on their opinion that the experimental studies providing the basis for the more stringent WHO recommendations were unrealistic models of clinical settings and that the risk of iatrogenic Creutzfeldt-Jakob disease (CJD) transmission must be very small, considering the small number of proven cases of CJD convincingly linked to documented exposures to contaminated neurosurgical instruments, as well as on an understandable concern that harsh chemical and heat treatments may damage surgical instruments. Although we agree that some prion inactivation studies were not ideal in mimicking the actual situations that occur in hospital settings, their findings provide reason for caution in accepting the less stringent sterilization options recommended by Rutala and Weber,' which we consider to be suboptimal and more likely to fail than those recommended by the WHO consultation.
Some recent studies designed to model hospital conditions more realistically have confirmed this caution. Yan et al ${ }^{3}$ used an inactivation model designed to closely mimic hospital conditions by contaminating steel wires with a laboratory prion strain (263K hamster-adapted scrapie agent); they concluded that initial enzymatic cleaning followed by steam sterilization at $134^{\circ} \mathrm{C}$ for 18 minutes, as well as a number of other decontamination regimens, did not completely inactivate TSE infectivity. Observations of analogous regimens by others yielded similar results. ${ }^{4,5}$ Yan et $\mathrm{al}^{3}$ also concluded that even the combination of $\mathrm{NaOH}$ and autoclaving at $134^{\circ} \mathrm{C}$ for 18 minutes may not be completely effective, so that decontamination protocols combining chemical and heat inactivation should be carefully selected and experimentally validated. ${ }^{3}$

Furthermore, we believe that the paucity of reported cases of iatrogenic TSE attributed to contaminated surgical instruments processed by conventional techniques may not be as reassuring as Rutala and Weber ${ }^{1}$ assert. Because of the long incubation periods of prion diseases, especially after low-dose exposures typical of cross-contamination events, identifying a surgical procedure that occurred many years before disease onset and then recognizing a causal link with an instrument contaminated by tissues of an index patient with a TSE oreven more difficult-a person silently incubating a TSE are extremely challenging. ${ }^{6}$ The Centers for Disease Control and Prevention (CDC) has participated in investigations of suspected CID transmissions by means of neurosurgical instruments in which results were inconclusive because poor record keeping and closure of hospitals made retrieval and adequate review of records impossible. ${ }^{7}$ The absence of incontrovertible epidemiologic evidence of CJD transmitted by contaminated surgical instruments that were reprocessed using current standard techniques does not prove that such transmission has not occurred.

On the basis of an estimate of mean infectivity likely to be present in human brains, Rutala and Weber ${ }^{1}$ concluded that their recommended method can be expected to eliminate an infecting dose of a human-derived TSE agent from a contaminated surface; however, that estimate may not apply in scenarios in which amounts of infectivity substantially exceed the mean value. Furthermore, others have reported that infectivity in brain tissue from a patient with CJD was even more heat-resistant than infectivity of the laboratory strain of rodent-adapted scrapie agent used as a model by Yan et al. ${ }^{3,8}$ The demonstrated resistance of TSE agent infectivity in tissue macerates to heat inactivation is extreme and unique among pathogens. ${ }^{9,10}$ Taken together, repeated failures in the laboratory of procedures similar to those suggested by Rutala and Weber ${ }^{1}$ to decontaminate prions justify continued caution before accepting the methods as sufficiently validated to recommend for use in clinical practice.

Readers should be aware that several prion disease experts 
at the CDC, the US Food and Drug Administration, the National Institutes of Health, and the United Kingdom National CJD Surveillance Unit, with other prominent prion disease researchers, continue to endorse using the more stringent decontamination protocols recommended by the WHO to decontaminate instruments that have had direct contact with both TSE high-infectivity and low-infectivity tissues. These recommended protocols are currently outlined at the CDC website $^{11}$ and involve the use of combined chemical and heat treatments as a preliminary decontamination step, followed by thorough cleaning and routine sterilization (Table 1). It is crucial to keep surgical instruments moist by immersing them in liquid to avoid air drying during the surgical procedure, not just after use, as recommended by Rutala and Weber. ${ }^{1}$ Current understanding of TSE high-infectivity and low-infectivity tissues has been summarized and periodically updated by the WHO. ${ }^{12}$ The most recent tables were updated in $2010 .^{13}$

We agree with Rutala and Weber ${ }^{1}$ that some materials cannot be subjected to such procedures. However, as long as proper precautions are followed, many reusable high-quality stainless steel surgical instruments should tolerate the more stringent WHO-recommended decontamination methods outlined in Table 1. The use of $\mathrm{NaOH}$ is preferable to $\mathrm{NaOCl}$, because it is much less corrosive to surgical instruments. Appropriate containment pans and lids have been shown to prevent damaging escape of $\mathrm{NaOH}$ into the autoclave cham-

TABLe 1. Prion Decontamination Protocols for Reusable Surgical Instruments (Single-Use Disposable Instruments Preferred When Feasible)

World Health Organization-recommended procedures

Immerse in $1 \mathrm{~N} \mathrm{NaOH}$ and heat in a gravity displacement autoclave at $121^{\circ} \mathrm{C}$ for 30 minutes in an appropriate container (see text, warnings, and references). Clean and sterilize by conventional means.

Immerse in $1 \mathrm{~N} \mathrm{NaOH}$ or $\mathrm{NaOCl}(20,000 \mathrm{ppm})$ for 1 hour. Transfer into water and autoclave (gravity displacement) at $121^{\circ} \mathrm{C}$ for 1 hour. Clean and sterilize by conventional means.

Immerse in $1 \mathrm{~N} \mathrm{NaOH}$ or $\mathrm{NaOCl}(20,000 \mathrm{ppm})$ for 1 hour. Rinse instruments with water, transfer to open pan and autoclave at $121^{\circ} \mathrm{C}$ (gravity displacement) or at $134^{\circ} \mathrm{C}$ (porous load) for 1 hour. Clean and sterilize by conventional means.

Warnings

$\mathrm{NaOH}$ should not be autoclaved in aluminum containers or in contact with aluminum.

Some poor-quality stainless steel instruments may be corroded by exposures to $\mathrm{NaOH}$ solutions; many metal instruments are corroded by exposures to $\mathrm{NaOCl}$ solutions.

Autoclave containers should have rims and lids designed to allow $\mathrm{NaOH}$ condensates to collect and drip back into the pan.

$\mathrm{NaOH}$ solutions are very caustic when hot and should be allowed to cool close to ambient temperature before handling using appropriate precautions. ber (Brown and Merritt ${ }^{14}$ and Brown et al ${ }^{15}$ ). Until more robust data about reliable inactivation of TSE infectivity become available, it remains prudent to use the more stringent decontamination methods shown in Table 1 to inactivate prions on contaminated surgical instruments.

\section{ACKNOWLEDGMENTS}

Potential conflicts of interest. All authors report no conflicts of interest relevant to this article.

Ermias D. Belay, MD;

Lawrence B. Schonberger, MD, MPH; Paul Brown, MD; Suzette A. Priola, PhD; Bruce Chesebro, MD; Robert G. Will, MD; David M. Asher, MD

From the Centers for Disease Control and Prevention, Atlanta, Georgia (E.D.B., L.B.S.); the National Institutes of Health, Hamilton, Montana (S.A.P. and B.C.); the US Food and Drug Administration, Silver Spring, Maryland (D.M.A.); Commissariat à l'Energie Atomique, France (P.B.); and the United Kingdom National Creutzfeldt-Jakob Disease Surveillance Unit, Edinburgh, Scotland (R.G.W.).

Address reprint requests to Ermias D. Belay, MD, 1600 Clifton Road, Atlanta, Georgia 30333 (EBelay@cdc.gov).

The findings and conclusions in this letter are those of the authors, have not been formally disseminated, and should not be construed to represent any agency determination or policy.

Infect Control Hosp Epidemiol 2010; 31(12):1304-1306

(C) 2010 by The Society for Healthcare Epidemiology of America. All rights reserved. 0899-823X/2010/3112-0018\$15.00. DOI: 10.1086/657579

\section{REFERENCES}

1. Rutala WA, Weber DJ. Guideline for disinfection and sterilization of prion-contaminated medical instruments. Infect Control Hosp Epidemiol 2010;31:107-117.

2. World Health Organization (WHO). Infection control guidelines for transmissible spongiform encephalopathies. Geneva, Switzerland: WHO; 2000. http://www.who.int/csr/resources/publications/bse/whocdscsraph 2003.pdf. Accessed March 24, 2010.

3. Yan ZX, Stitz L, Heeg P, Pfaff E, Roth K. Infectivity of prion protein bound to stainless steel wires: a model for testing decontamination procedures for transmissible spongiform encephalopathies. Infect Control Hosp Epidemiol 2004;25:280-283.

4. Jackson GS, McKintosh E, Flechsig E, et al. An enzyme-detergent method for effective prion decontamination of surgical steel. J Gen Virol 2005; 86(pt 3):869-878.

5. Pomeroy K, Brown S, Woods T, Asher D. Decontamination of surfaces exposed to the infectious agents of TSEs. Paper presented at: Prion 2009; September 2009; Thessaloniki-Chalkidiki, Greece. Abstract P.2.39; p 81.

6. Keeler N, Schonberger LB, Belay ED, Sehulster L, Turabelidze G, Sejvar JJ. Investigation of a possible iatrogenic case of Creutzfeldt-Jakob disease after a neurosurgical procedure. Infect Control Hosp Epidemiol 2006;27: 1352-1357.

7. Stricof RL, Lillquist PP, Thomas N, Belay ED, Schonberger LB, Morse DL. An investigation of potential neurosurgical transmission of Creutzfeldt-Jakob disease: challenges and lessons learned. Infect Control Hosp Epidemiol 2006;27:302-304.

8. Peretz D, Supattapone $S$, Giles $K$, et al. Inactivation of prions by acidic sodium dodecyl sulfate. $J$ Virol 2006;80:322-331.

9. Brown P, Rau EH, Lemieux P, Johnson BK, Bacote AE, Gajdusek DC. Infectivity studies of both ash and air emissions from simulated incin- 
eration of scrapie-contaminated tissues. Environ Sci Technol 2004;38: 6155-6160.

10. Taylor DM. Preventing accidental transmission of human transmissible spongiform encephalopathies. Br Med Bull 2003;66:293-303.

11. Centers for Disease Control and Prevention (CDC). Infection control practices: CJD (Creutzfeldt-Jakob disease, classic). http://www.cdc.gov/ ncidod/dvrd/cjd/qa_cjd_infection_control.htm

12. World Health Organization (WHO). Guidelines on tissue infectivity distribution in transmissible spongiform encephalopathies. Geneva, Switzerland: WHO; 2006.http://www.who.int/bloodproducts/TSEREPORT -LoRes.pdf. Accessed March 24, 2010.

13. World Health Organization. WHO tables on tissue infectivity distribution in transmissible spongiform encephalopathies. http://www.who.int/ bloodproducts/tablestissueinfectivity.pdf. Accessed October 18, 2010.

14. Brown SA, Merritt $K$. Use of containment pans and lids for autoclaving caustic solutions. Amer J Infect Control 2003;31(4):257-260.

15. Brown SA, Merritt K, Woods TO, Busick DN. Effects on instruments of the World Health Organization-recommended protocols for decontamination after possible exposure to transmissible spongiform encephalopathy-contaminated tissue. I Biomed Mater Res B Appl Biomater 2005; $72(1): 186-190$.

\section{Reply to Belay et al}

To the Editor-We thank Belay et $\mathrm{al}^{1}$ for their opinions. We agree with their statement that "conflicting TSE decontamination protocols have been proposed in the past because of concerns about the unusual resistance of infectivity to conventional sterilization methods, the paucity of appropriate laboratory and epidemiologic data, and the difficulties associated with conducting inactivation studies more relevant

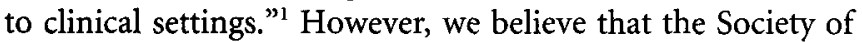
Healthcare Epidemiology of America (SHEA) guideline titled "Guideline for Disinfection and Sterilization of Prion-Contaminated Medical Instruments" provides the best recommendations on the basis of a systematic review of the relevant scientific literature. ${ }^{2}$ The guideline has been reviewed by multiple experts and approved by the SHEA Guideline Committee and the SHEA Board of Directors.

We wish to comment on several of the points raised in this letter. ${ }^{1}$ First, the authors rely on the World Health Organization (WHO) Consensus Conference report published in 1999 as the basis for their current recommendations. ${ }^{3}$ We have several concerns with using this as the basis for current recommendations: (1) the WHO report is not an evidencebased guideline, (2) the WHO recommendations do not list any scientific studies to support individual recommendations, and (3) the WHO report is now more than 10 years old, and in the past 10 years more scientific studies on disinfection and sterilization of prions have been published than in the period before 1999. In their letter, Belay et al ${ }^{1}$ list only 3 of the 6 disinfection protocols recommended by the WHO in 1999. A detailed explanation based on the scientific literature for the exclusion of specific sterilization protocols is not provided. This highlights the problem of providing non-refer- ence-based recommendations. In fact, we note that the first option in the SHEA Guideline for disinfection of prion-contaminated instruments (ie, autoclaving at $134^{\circ} \mathrm{C}$ for 18 minutes), which was criticized by Belay et $\mathrm{al},{ }^{1}$ is a method specifically recommended in the WHO 1999 publication. ${ }^{3}$

Second, we agree that a perfect test system for assessing the effectiveness of methods for disinfection of medical devices contaminated by prions does not exist. In the past 30 years, we have had the opportunity to evaluate the activity of disinfectants and sterilization processes against microorganisms that cause healthcare-associated infections. We and others have repeatedly demonstrated that all disinfection or sterilization processes can be made to fail by altering the test method or parameters for disinfection and/or sterilization. ${ }^{4-11}$ In fact, we can make any Environmental Protection Agencyregistered disinfectant and any US Food and Drug Administration-cleared sterilization process fail to inactivate even easy-to-kill microorganisms, such as bacteria, on the basis of the methodology used. It is crucial, especially when assessing the efficacy of protocols for the disinfection of prions, that one place greater weight on studies using disinfection protocols representative of current and standard instrument reprocessing procedures. Most studies of prion disinfection were conducted under conditions that do not reflect the reprocessing procedures in a clinical setting. For example, "lumps" of brain tissue weighing 50 or $375 \mathrm{mg}$ were used, and investigators found, not surprisingly, that the larger tissue samples were more refractory to heat inactivation. ${ }^{12}$ However, surgical instruments processed in the central processing departments of 5 hospitals were shown to have a median protein contamination level of only 8-91 $\mu \mathrm{g}$ per instrument. ${ }^{13}$

Third, we place more weight than Belay et $\mathrm{al}^{1}$ do on the lack of reports of prion transmission due to failure of disinfection and sterilization. Since 1976, there have been no documented cases of Creutzfeldt-Jakob disease (CJD) transmission due to a contaminated surgical instrument.

Fourth, we have carefully reviewed the articles by Yan et al..$^{14,15}$ We note that Yan et $\mathrm{al}^{15}$ in one article showed complete inactivation of TSE infectivity using $134^{\circ} \mathrm{C}$ for 18 minutes without cleaning, whereas the $1 \mathrm{M}$ sodium hydroxide immersion for 24 hours plus $134^{\circ} \mathrm{C}$ for 18 minutes failed to provide complete prion inactivation. In the other article, Yan et $\mathrm{al}^{14}$ showed that both processes failed to achieve complete prion inactivation, but that $134^{\circ} \mathrm{C}$ for 18 minutes without cleaning was more effective than the $1 \mathrm{M}$ sodium hydroxide immersion for 24 hours plus $134^{\circ} \mathrm{C}$ for 18 minutes. Yan et $\mathrm{al}^{14,15}$ also showed that certain enzymatic cleaners dramatically reduced the effectiveness of steam sterilization using $134^{\circ} \mathrm{C}$ for 18 minutes for prion inactivation, whereas an alkaline detergent and steam sterilization resulted in complete prion inactivation. The SHEA guideline mentions that some studies have revealed excellent but not complete inactivation of the test prions with steam sterilization only and the same result for use of $\mathrm{NaOH}$ and steam sterilization. ${ }^{2}$ This is likely explained by methodological differences between the studies, 